\title{
EL DESARROLLO DE CANCÚN, ANALIZADO DESDE LA TEORÍA DE LOS SISTEMAS COMPLEJOS
}

\author{
Autores \\ Romano Segrado Pavón \\ Master en Estudios Turísticos \\ Universidad de Qintana Roo \\ México \\ romano@uqroo.mx \\ Alfonso González Damián \\ Master en Administración \\ Dr. en Cs. Sociales y Politicas \\ Universidad de Quintana Roo \\ gonzalezd@uqroo.mx

\section{Lucinda Arroyo Arcos} \\ Universidad de Qintana Roo \\ México \\ larroyo@uqroo.mx

\section{Alejandro Palafox Muñoz \\ Master en Estudios Turísticos \\ Universidad de Quintana Roo \\ palafox@uqroo.mx}

\section{RESUMEN}

Se analizó el sistema productivo del Municipio Benito Juárez, desde la teoría de "sistemas complejos", aplicando la metodología propuesta por García (2000), quien propone diferentes niveles de análisis (local, regional-nacional e, internacional), para cada sistema social, respetando los principios de organización y evolución. La investigación se basó en fuentes documentales (libros e Internet).

Se construyó un modelo para representar la realidad actual y cuya confirmación satisfactoria sólo puede basarse en su capacidad de explicar el funcionamiento de los hechos, sin caer en la generalización de que "todo interactúa con todo". Como resultados, se determinó que Cancún y su principal actividad económica -el turismo- forma parte de un sistema complejo cuya evolución responde a la dinámica propia de esta clase de sistemas; que funciona como una totalidad organizada y, que los cambios en la actividad turística están en correspondencia con cambios significativos en el subsistema económico.

Palabras clave: Cancún, Sistemas complejos, turismo 


\title{
A COMPLEX SYSTEMS THEORY BASED ANALYSIS OF THE DEVELOPMENT OF CANCÚN, MEXICO
}

\author{
Authors \\ Romano Segrado Pavón \\ Master en Estudios Turísticos \\ Universidad de Qintana Roo \\ México \\ romano@uqroo.mx \\ Alfonso González Damián \\ Master en Administración \\ Dr. en Cs. Sociales y Politicas \\ Universidad de Quintana Roo \\ gonzalezd@uqroo.mx

\section{Lucinda Arroyo Arcos \\ Universidad de Qintana Roo \\ México \\ larroyo@uqroo.mx} \\ Alejandro Palafox Muñoz \\ Master en Estudios Turísticos \\ Universidad de Quintana Roo \\ palafox@uqroo.mx
}

\begin{abstract}
This paper reports a study of the productive sector of the economy of the municipality of Benito Juarez (Cancún) using García’s (2000) methodology. García proposes applying three different levels of analysis (local, regional/national and international) to each social system studied. The analysis takes into account both the way these systems started and how they have developed to the present day. The data comes mainly from documental sources, including internet.

The model developed was validated through its capacity to explain the current economic situation. It is shown that tourism, the principle economic activity, has evolved to form part of a complex system which works as a whole and reflects changes in the economic subsystem.
\end{abstract}

Key words: Cancun, complex systems, tourism 


\section{INTRODUCCIÓN}

La teoría general de sistemas surgió a mediados del siglo XX, y aunque algunos científicos ya pregonaban dicho concepto desde finales del siglo XIX, el reduccionismo total y el conflicto marxismo-capitalismo aún estaban demasiado en auge como para que se escuchasen nuevas voces.

Básicamente, la teoría general de sistemas implica que toda entidad o sistema forma parte de otro sistema mayor, con su correspondiente organización de entrada, procesamiento, salida y retroalimentación. Como propuesta teórica surgió con la intención de ser una alternativa a los enfoques analítico-mecánicos que fragmentaban la realidad, dividiendo los fenómenos en partes, tanto como fuera posible, bajo la premisa de que todo problema puede ser solucionado separando sus partes fundamentales y analizándolas individualmente.

El enfoque sistémico como aproximación teórica de la realidad ofrece algunas ventajas frente a los enfoques analítico-mecánicos estructuralistas, entre otras:

1. Pone en el centro de la teoría, no a la unidad, sino al todo, que no se reduce a la suma de sus partes.

2. Concibe la noción de sistema, como no formal o ambigua.

3. Se sitúa en un nivel transdisciplinario (Morín, 2001).

La Teoría General de Sistemas, construida a partir de la noción de sistemas, ha sido utilizada como punto de partida para explicar al turismo al observarlo como un sistema con entradas y salidas, como un fenómeno socio - económico y espacial, en el cual se interrelacionan diferentes elementos. Al respecto, la SECTUR (2000) afirma que el turismo como objeto de estudio, puede ser abordado desde diferentes disciplinas científicas, siendo muy útil un enfoque transdisciplinario como la teoría de sistemas, para estudiarlo integralmente.

Con esta idea, diversos autores propusieron modelos de sistemas aplicados a la actividad turística, cada uno de enfocado al análisis de sus elementos estructurales. Raimundo Cuervo (1967), propuso un sistema turístico a partir de dos elementos, la oferta de servicios y las demandas de los viajeros. Neil Leiper (1990), identifica los elementos del sistema desde un ángulo espacio-territorial, región de origen, de destino y ruta de tránsito, un elemento dinámico, el flujo de turistas y un entorno con el cual interactúa el sistema. Posteriormente Sergio Molina (1991) propuso otra forma de comprender el sistema, al que incorporó los conceptos de superestructura, estructura e infraestructura. Gunn (1994), propone un sistema centrado en la oferta y la demanda, Mill y Morrison (1985) proponen un sistema centrado en el marketing del turismo, estos dos trascienden 
la visión mecánica del sistema y se centran en la dinámica que lo hace funcionar, sin embargo suponen un conjunto de relaciones elementales entre los elementos y desdeñan la integración del sistema con su entorno.

Aunque sea difícil abarcar todas las aproximaciones se puede afirmar que algo común a todos los modelos citados es que suponen un conjunto de elementos relacionados entre sí que interactúan, pero tales elementos son separados para su examen individual aunque en realidad estén ligados y la interrelación sea lo que permita su verdadero entendimiento (Cooper, 1997), por lo que se puede afirmar que estas propuestas son útiles, "para profundizar en el análisis de cuestiones puntuales, pero insuficientes para comprender y explicar la totalidad en la que se dan los componentes que se analizan" (Ander-Egg, 2001: 107).

Enfocado así, el análisis intenta explicar el fenómeno por medio de la división y reducción de los componentes que originan la actividad del turismo, y que hasta ahora no ha permitido un entendimiento amplio del fenómeno, por ello más recientemente diversos autores se han aproximado al turismo con enfoques alternativos al tradicional de la teoría general de sistemas, algunos de ellos incorporando el concepto de complejidad como característica inherente del sistema turístico, ya que "existen circunstancias que están llevando a la necesidad de desarrollar un pensamiento complejo (...). Esto exige abrirse al pensamiento de la complejidad de lo real, forjar un pensamiento capaz de pensar esa complejidad” (Ander-Egg, 2001: 93).

En este sentido, el sistema turístico posee una disposición, estructuración, y elementos se relacionan entre sí para efectuar operaciones de entrada, procesamiento, salida y retroalimentación, características estructurales del sistema; se puede afirmar que además, el sistema turístico tiene información oculta entre sus elementos y relaciones, lo que le confiere la complejidad al sistema, la cual se incrementa exponencialmente mientras más elementos incorpora, ya que las interrelaciones y comunicaciones entre los mismos crearán sinergia y mutua dependencia, esta última característica es la que propicia que el sistema no sea descomprimible (García, 2000). En sistemas mecánicos, no complejos, por ejemplo: en una computadora se sabe como funciona en cada una de sus partes y sí se desarma y vuelve a armar, nada cambia y las relaciones se mantienen. En un sistema complejo, en cambio, existen variables ocultas cuyo desconocimiento nos impide analizar el sistema con precisión. Por ejemplo, una comunidad y sus actividades económicas no pueden ser separadas y sí lo fuesen, al ser reintegradas, no se tendría la certeza de que las relaciones originales fuesen las mismas o de que existan otras nuevas, es decir que el sistema no es descomprimible. Así pues, un sistema complejo posee más información que la que da cada parte individualmente. Es por esta razón, que si se quiere comprender mejor el funcionamiento de un sistema, el análisis de su estructura, desde un punto de vista mecánico, convencional, puede resultar limitado, en tanto que 
un enfoque desde el ángulo de la complejidad tiene la probabilidad de aportar una mayor comprensión.

Como sistema de análisis no descomprimible, el turismo, según Uriel y Monfort (citado por Muñoz, 2005) "se distingue por la dificultad de delimitar las ramas que le dan cuerpo, como consecuencia del carácter mixto de la oferta turística y de la práctica imposibilidad de cuantificar algunos elementos que también forman parte de la misma; bien sea el paisaje, la cultura o el medio natural (...)", es decir que como fenómeno complejo, interactúa con las dimensiones económicas, sociales, políticas, culturales, naturales, etc. y no puede ser encasillado en un sector, por lo que requiere ser analizado de forma amplia e integral, ya que posee unas características sui generis que le son inherentes. Estas características son, según Uriel y Monfort (Ibid):

1. La ambigüedad en la delimitación del área de análisis

2. Las múltiples y complejas interrelaciones existentes entre los elementos constitutivos del hecho turístico.

3. La heterogeneidad de los sectores o actividades.

4. La imposibilidad de definir el sistema de elaboración del producto turístico.

5. Unas actividades de naturaleza dispar, con estrategias y diseños heterogéneos.

6. El producto se encuentra alejado del consumidor

7. El demandante se desplaza hacia la oferta

8. La dependencia de los recursos naturales en los que se apoya la oferta turística

9. Fuerte presencia de las instituciones públicas locales

10. Dependencia al contexto social y político donde se desarrolla.

Por esto, si se desea lograr un análisis que explique el fenómeno y refleje la realidad, es necesario adaptarse a nuevos paradigmas. En el caso del turismo, además de la heterogeneidad, la característica determinante de un sistema complejo es la interdefinibilidad y mutua dependencia de las funciones que cumplen dichos elementos dentro del sistema total y esta característica excluye la posibilidad de obtener una explicación de la realidad del funcionamiento del sistema por la simple adición de estudios sectoriales correspondientes a cada uno de los elementos (García, 1994). Esto significa que el resultado no puede ser atribuido a cada componente por si sólo, sino que es consecuencia de la acción cooperativa o antagónica entre ellos.

Con esta base, se propuso la teoría de sistemas complejos como una alternativa a los enfoques analítico-mecánicos que fragmentaban la realidad, dividiendo los fenómenos en partes, tanto como fuera posible, para analizarlos separadamente, bajo la premisa de que todo problema puede ser solucionado separando sus partes fundamentales y analizándolas individualmente. Esto fue útil, "para profundizar en el análisis de cuestiones puntuales, pero insuficiente para comprender y explicar la totalidad en la que 
se dan los componentes que se analizan” (Ander-Egg, 2001: 107).

Con el enfoque de la teoría de "sistemas complejos", el turismo es considerado como un subsistema del sistema total, lo cual permite mostrar que su papel en la producción encuentra su explicación en las transformaciones y cambios sufridos por el sistema, a través de sucesivas reorganizaciones en las que intervinieron diversos factores. Se han identificado las condiciones macroeconómicas y sociopolíticas de dichos cambios, y el rol de las políticas nacionales, para determinar la existencia de un sistema complejo.

Una definición muy sencilla de un sistema "complejo", es la de un gran número de elementos los cuales interactúan entre sí (Bar-Yam, 1997). La funcionalidad global del sistema se da precisamente por las interacciones, y por lo tanto no se encontrará tal funcionalidad si uno observa sólo a unos cuantos elementos. En resumen, se deben observar las relaciones del sistema, no sus elementos. Sobre esta misma idea coinciden Leff (1986), García (1994), Luhmann (1997), y Morin (2001). Al centrarse en las relaciones, al atender a la dinámica del sistema antes que a su estructura, al reconocer lo variable por sobre lo estático, se da cabida a lo aleatorio, a lo caótico, a la incertidumbre, con lo que la mirada se enfrenta a la necesidad de abandonar la búsqueda de la certeza y la objetividad, o al menos a mantenerla en suspenso.

Los trabajos de Farrell y Twining-ward (2003), Jiménez (2005) y Osorio (2010) abren la puerta a la complejidad en el análisis sistémico del turismo, el primero plantea la necesidad de comprender al turismo como un sistema complejo adaptativo, complejo dado que involucra relaciones, variables y procesos que van más allá de la denominada industria turística y adaptativo dado que como otros sistemas, muestra una tendencia natural a la entropía pero que a través de flujos de energía es resuelta mediante un proceso de auto-organización; el segundo introduce la idea de la simultaneidad de funciones para cada elemento del sistema y con otros sistemas, lo que resulta en una red global de subsistemas turísticos o sistema turístico global. Osorio (2010) por su parte, da un paso más hacia la complejidad y propone un reenfoque en el análisis del turismo como sistema al análisis del turismo como diferenciación funcional en el sistema social y destaca la necesidad de comprender la diferenciación mediante una observación de segundo orden centrada en la operación contingente y en el acoplamiento estructural de los sistemas funcionales autopoiéticos en la sociedad compleja.

\section{MÉTODO}

La investigación sistémica tiene una amplia gama de propuestas de investigación, más aún para el análisis de políticas públicas, que es un campo de conocimiento multidisciplinario. Velasco (2005: 170) argumenta que en los últimos cincuenta años, 
las aproximaciones teóricas a la investigación y análisis de políticas públicas se puede ordenar en un continuo con dos extremos: 1) análisis en profundidad alguno de los elementos de una política pública (análisis de la formulación de los problemas que se abordan, de la implantación de los programas o de la evaluación) o 2) análisis de las relaciones de una política concreta con un entorno político más amplio.

Por su parte, Hogwood y Gunn (Citados por Velasco, 2005) proponen distinguir entre diferentes "variedades en el análisis de políticas públicas" considerando los siguientes:

1. Análisis de contenidos: aquellos estudios que analizan la génesis y el desarrollo de políticas concretas.

2. Análisis del proceso: investigaciones centradas en las etapas del ciclo de las políticas y en factores de influencia de cada una de ellas.

3. Estudio de productos: estudios que toman una política pública concreta como variable dependiente y estudian las relaciones externas.

4. Evaluación: investigaciones que valoran el proceso o sus resultados desde variables predeterminadas.

5. Información para la mejora del proceso de toma de decisiones.

6. Análisis para mejorar los procesos de elaboración de políticas.

7. Defensa de determinadas opciones o presión en el proceso o en las políticas.

Estos siete tipos de análisis pueden agruparse en dos categorías, aquellos centrados en el análisis del proceso y aquellos centrados en el análisis para el proceso (Ver tabla 2).

Tabla n 2. Tipos de análisis de políticas públicas

\begin{tabular}{|l|l|l|l|l|l|l|}
\hline $\begin{array}{l}\text { Estudio del } \\
\text { conteni- } \\
\text { do de la } \\
\text { política }\end{array}$ & $\begin{array}{l}\text { Estudio del } \\
\text { proceso de } \\
\text { la política }\end{array}$ & $\begin{array}{l}\text { Estudio de } \\
\text { los pro- } \\
\text { ductos del } \\
\text { proceso }\end{array}$ & Evaluación & $\begin{array}{l}\text { Informa- } \\
\text { ción para } \\
\text { el proceso } \\
\text { decisorio }\end{array}$ & $\begin{array}{l}\text { Process } \\
\text { advocacy }\end{array}$ & $\begin{array}{l}\text { Defensa de } \\
\text { políticas }\end{array}$ \\
\hline & & & & $\begin{array}{l}\text { Análistas } \\
\text { como } \\
\text { actores } \\
\text { politicos }\end{array}$ & $\begin{array}{l}\text { Politicos } \\
\text { como ana- } \\
\text { listas }\end{array}$ \\
\hline
\end{tabular}

Fuente: Hogwood y Gunn, 1981

En este estudio se pretendió abordar la planificación turística como política pública y analizarla como "signos reveladores" de diferentes proposiciones jerárquicas mayores, que constituirían los marcos de aproximación (Rein y Schön, 1993). Esto es, comprender a la planificación turística como resultado del funcionamiento de un 
sistema socio-económico integrado desde el nivel internacional al municipal o local, para analizar la génesis, el desarrollo y los contenidos, en relaciones causa-efecto.

Al considerar los argumentos expuestos, se tomó como punto de partida a las primeras acciones públicas diseñadas para el turismo, tal y como hoy se entiende, en el territorio quintanarroense, con la aparición del polo turístico de Cancún a principios de la década de los setenta del siglo XX. Asimismo, con el fin de concentrar la atención en la zona estudiada, se decidió centrar la atención, sin excluir otros instrumentos de planificación, en los documentos publicados por el gobierno federal o estatal en los que expresamente se hiciera mención al turismo en Cancún o su área de influencia.

El proceso de indagación abarcó las siguientes etapas:

1. Búsqueda de información bibliográfica-documental y selección de los instrumentos a analizar.

2. Análisis del contendido de los instrumentos en las siguientes categorías:

a. Vínculos entre documentos

b. Nivel jerárquico en el que aparece el turismo

c. Inclusión de políticas expresas para el turismo en Cancún

d. Identificación de períodos y contextos sociopolíticos correspondientes

e. Identificación de proposición teórica subyacente

3. Conclusiones a partir del análisis de contenido e identificación de la génesis y transformaciones de la planificación turística en la zona de estudio.

Tras una primera revisión, se agrupó a los instrumentos en seis, en función de su ámbito de aplicación y de su área de atención: 1) Instrumentos marco, 2) Instrumentos nacionales específicos, 3) Instrumentos estatales marco, 4) Instrumentos estatales específicos, 5) Instrumentos locales específicos 6) Instrumentos parcialmente vinculados. Entre ellos se seleccionó a los documentos listados en la tabla $n^{\circ} 3$ para realizar el análisis.

Si bien existen múltiples instrumentos de planificación que abordan de algún modo a la actividad turística, se han seleccionado algunos de ellos, por las características de la región y del turismo que se pretende desarrollar en ella, en términos de la propia política turística del Estado. En el estudio se identificaron algunos instrumentos tanto de ámbito nacional como local que dictan política específica sobre el uso de los recursos naturales y del patrimonio histórico.

Tabla $n^{\circ}$ 3. Instrumentos de planificación turística seleccionados

\begin{tabular}{|l|l|l|l|l|}
\hline Plan & Nombre del instrumento & Titular & $\begin{array}{l}\text { Dependencia a } \\
\text { cargo }\end{array}$ & $\begin{array}{l}\text { Tipo de } \\
\text { instrumento }\end{array}$ \\
\hline PND 00-06 & $\begin{array}{l}\text { Plan Nacional de } \\
\text { Desarrollo }\end{array}$ & Vicente Fox Quesada & $\begin{array}{l}\text { Ejecutivo } \\
\text { Federal }\end{array}$ & 1 \\
\hline
\end{tabular}




\begin{tabular}{|c|c|c|c|c|}
\hline PND 06 & \begin{tabular}{|l|} 
Planes Nacionales de \\
desarrollo 06
\end{tabular} & $\begin{array}{l}\text { Felipe Calderón } \\
\text { Hinojosa }\end{array}$ & \begin{tabular}{|l|} 
Ejecutivo \\
Federal
\end{tabular} & 1 \\
\hline PND 76-82 & \begin{tabular}{|l|} 
Plan Nacional de \\
Desarrollo \\
\end{tabular} & José López Portillo & \begin{tabular}{|l|} 
Ejecutivo \\
Federal \\
\end{tabular} & 1 \\
\hline PND 82-88 & $\begin{array}{l}\text { Plan Nacional de } \\
\text { Desarrollo }\end{array}$ & $\begin{array}{l}\text { Miguel de la Madrid } \\
\text { Hurtado }\end{array}$ & $\begin{array}{l}\text { Ejecutivo } \\
\text { Federal } \\
\end{array}$ & 1 \\
\hline PND 88-94 & $\begin{array}{l}\text { Plan Nacional de } \\
\text { desarrollo }\end{array}$ & $\begin{array}{l}\text { Carlos Salinas de } \\
\text { Gortari }\end{array}$ & \begin{tabular}{|l|} 
Ejecutivo \\
Federal \\
\end{tabular} & 1 \\
\hline PND 94-00 & \begin{tabular}{|l|}
$\begin{array}{l}\text { Plan Nacional de } \\
\text { desarrollo }\end{array}$ \\
\end{tabular} & $\begin{array}{l}\text { Ernesto Zedillo } \\
\text { Ponce de León } \\
\end{array}$ & \begin{tabular}{|l} 
Ejecutivo \\
Federal \\
\end{tabular} & 1 \\
\hline PST 00-06 & $\begin{array}{l}\text { Programa Sectorial de } \\
\text { Turismo }\end{array}$ & $\begin{array}{l}\text { Leticia Navarro } \\
\text { Ochoa }\end{array}$ & SECTUR & 2 \\
\hline PST 06-12 & $\begin{array}{l}\text { Programa Sectorial de } \\
\text { Turismo }\end{array}$ & $\begin{array}{l}\text { Rodolfo Elizondo } \\
\text { Torres }\end{array}$ & SECTUR & 2 \\
\hline PST 76-82 & Plan de Turismo & $\begin{array}{l}\text { Guillermo Rossell de } \\
\text { la Lama }\end{array}$ & SECTUR & 2 \\
\hline PST 82-88 & $\begin{array}{l}\text { Programa Sectorial de } \\
\text { Turismo }\end{array}$ & $\begin{array}{l}\text { Antonio Enríquez } \\
\text { Savignac }\end{array}$ & SECTUR & 2 \\
\hline PST 88-94 & $\begin{array}{l}\begin{array}{l}\text { Programa Sectorial de } \\
\text { Turismo }\end{array} \\
\end{array}$ & \begin{tabular}{|l|} 
Carlos Hank \\
González \\
\end{tabular} & SECTUR & 2 \\
\hline PST 94-00 & $\begin{array}{l}\text { Programa Sectorial de } \\
\text { Turismo }\end{array}$ & \begin{tabular}{|l} 
Silvia Hernández \\
Enríquez
\end{tabular} & SECTUR & 2 \\
\hline PB 99-05 & $\begin{array}{l}\text { Plan Estatal de } \\
\text { Desarrollo 99-05 }\end{array}$ & $\begin{array}{l}\text { Joaquín Hendricks } \\
\text { Díaz }\end{array}$ & SEPLADER & 3 \\
\hline PED 05-11 & $\begin{array}{l}\text { Plan Estatal de } \\
\text { Desarrollo 05-11 }\end{array}$ & Félix González Canto & SEPLADER & 3 \\
\hline PED 81-87 & $\begin{array}{l}\text { Plan Estatal de } \\
\text { Desarrollo 82-88 }\end{array}$ & \begin{tabular}{|l} 
Pedro Joaquín \\
Coldwell
\end{tabular} & SEPLADER & 3 \\
\hline PED 87-93 & \begin{tabular}{|l} 
Plan Estatal de \\
Desarrollo 87-93
\end{tabular} & Miguel Borge Martín & SEPLADER & 3 \\
\hline PED 93-99 & \begin{tabular}{|l} 
Plan Estatal de \\
Desarrollo 93-99
\end{tabular} & Mario Villanueva & SEPLADER & 3 \\
\hline PGV 20-25 & $\begin{array}{l}\text { Plan Gran Visión 2000- } \\
2025\end{array}$ & $\begin{array}{l}\text { Orlando Arroyo } \\
\text { Marroquín } \\
\end{array}$ & CCE & 4 \\
\hline PS- 05-11 & $\begin{array}{l}\text { Programa Sectorial } \\
\text { Turismo }\end{array}$ & $\begin{array}{l}\text { Gabriela Rodríguez } \\
\text { Gálvez }\end{array}$ & SEDETUR & 4 \\
\hline PMCM & $\begin{array}{l}\text { Plan Maestro de la Costa } \\
\text { Maya }\end{array}$ & $\begin{array}{l}\text { John McCarthy } \\
\text { Sandland }\end{array}$ & FONATUR & 5 \\
\hline PRODESUR & $\begin{array}{l}\text { Programa de Desarrollo } \\
\text { Turístico del Sur de Q. } \\
\text { Roo }\end{array}$ & Cora Amalia Castilla & MPIO OPB & 5 \\
\hline 2001-2006 & $\begin{array}{l}\text { Programa Nacional de } \\
\text { Cultura }\end{array}$ & Sari Bermúdez & $\begin{array}{l}\text { CONACULTA } \\
\text { / NAH }\end{array}$ & 6 \\
\hline
\end{tabular}




\begin{tabular}{|l|l|l|l|l|}
\hline $\begin{array}{l}\text { PNMA 01- } \\
\text { 06 }\end{array}$ & $\begin{array}{l}\text { Programa Nacional de } \\
\text { Medio Ambiente y } \\
\text { Recursos Naturales } \\
\text { 2001-2006 }\end{array}$ & $\begin{array}{l}\text { Víctor Lichtinger } \\
\text { Waisman }\end{array}$ & SEMARNAT & 6 \\
\hline PNMA 06 & $\begin{array}{l}\text { Programa Nacional } \\
\text { de Medio Ambiente } \\
\text { y Recursos Naturales } \\
\text { 2001-2006 }\end{array}$ & $\begin{array}{l}\text { Juan Rafael Elvira } \\
\text { Quezada }\end{array}$ & SEMARNAT & 6 \\
\hline $\begin{array}{l}\text { POET } \\
\text { QROO }\end{array}$ & $\begin{array}{l}\text { Programa Ordenamiento } \\
\text { Territorial Q. Roo }\end{array}$ & $\begin{array}{l}\text { Francisco Javier Díaz } \\
\text { Carvajal }\end{array}$ & SEDUMA & 6 \\
\hline
\end{tabular}

Fuente: González, 2008.

De esta forma, el estudio está delimitado no por la extensión del tema, sino por la perspectiva globalizadora (Figura $n^{\circ} 1$ ) que, en su afán de visualizar el todo integral, hace desaparecer los detalles que importan al análisis minucioso, para detener la vista de los elementos importantes y sus relaciones destacadas, en un intento de esbozar el "todo integral” en sus líneas fundamentales (Rodríguez, 1999). Igualmente, ningún estudio puede abarcar la totalidad de las relaciones o de las condiciones de contorno dentro de un sistema complejo (aún en el supuesto de que tenga sentido hablar de tal "totalidad"). Siempre se necesita de criterios de selección (García, citado por Leff; 1986).

Para el caso del presente documento, el elemento que permitirá explicar la evolución del sistema turístico será el cambio o adaptación reorganizadora del sistema al acoplarse estructuralmente a cambios en su entorno. García (2000) sugiere tres niveles de análisis para explicar la evolución y organización de un sistema determinado:

Nivel I: Cambios que afectan al sistema por cambios en algunos de sus elementos e interrelaciones tales como cambios introducidos al sistema por modificaciones en las relaciones socioeconómicas y o en el medio físico.

Nivel II: Cambios introducidos en el sistema productivo (introducción de ideas, créditos, mano de obra, etc.) como resultado de cambios de políticas nacionales.

Nivel III: Se refiere a los cambios que ocurren en las políticas nacionales de desarrollo, debido a modificaciones en las políticas internacionales o de mercado.

En el caso de este documento la mirada se centra en dos subsistemas: el económico- social y el turístico, aunque el modelo final incluye los tres subsistemas vinculantes:

1. Subsistema Físico (SSF): suelo, agua, clima, ecosistemas naturales.

2. Subsistema Económico Social (SSES): grupos sociales, factores económicos, factores políticos y,

3. Subsistema Turístico (SST): atractivos, servicios, comunicación. Dentro de este subsistema, se abordaron los componentes más importantes de la actividad 
turística: hoteles, restaurantes, aviones, cruceros, mano de obra, migración, leyes, turistas, aunque este documento lo hace de forma general.

La delimitación histórica, para determinar períodos de análisis, se realizó con base en Romo (1995) y sus etapas del desarrollo económico, aunque también se ha considerado a otros autores, tales como Rostow (1961) Cordera (1979), Holder (1987), y los instrumentos presentados en la tabla $\mathrm{n}^{\circ} 3$, por lo que la finalmente, la delimitación muestra una estrecha relación con los sexenios presidenciales:

(i) Primer período: Crecimiento sin inflación (1956-1970)

(ii) Segundo período: Monetarismo (1971-1976)

(iii) Período de transición: Estancamiento con inflación (1976-1982)

(iv) Tercer período: Apertura de mercados (1983-actual)

Debido a los desfases en las escalas espaciales y temporales entre los procesos de distinto nivel no deberá buscarse una correspondencia estricta entre el comienzo y final de ellos, sino sus relaciones más relevantes (Becerra, 1997; Dobb, 1991).

Figura $n^{\circ} 1$. Perspectiva Global

\section{Sistema Global}

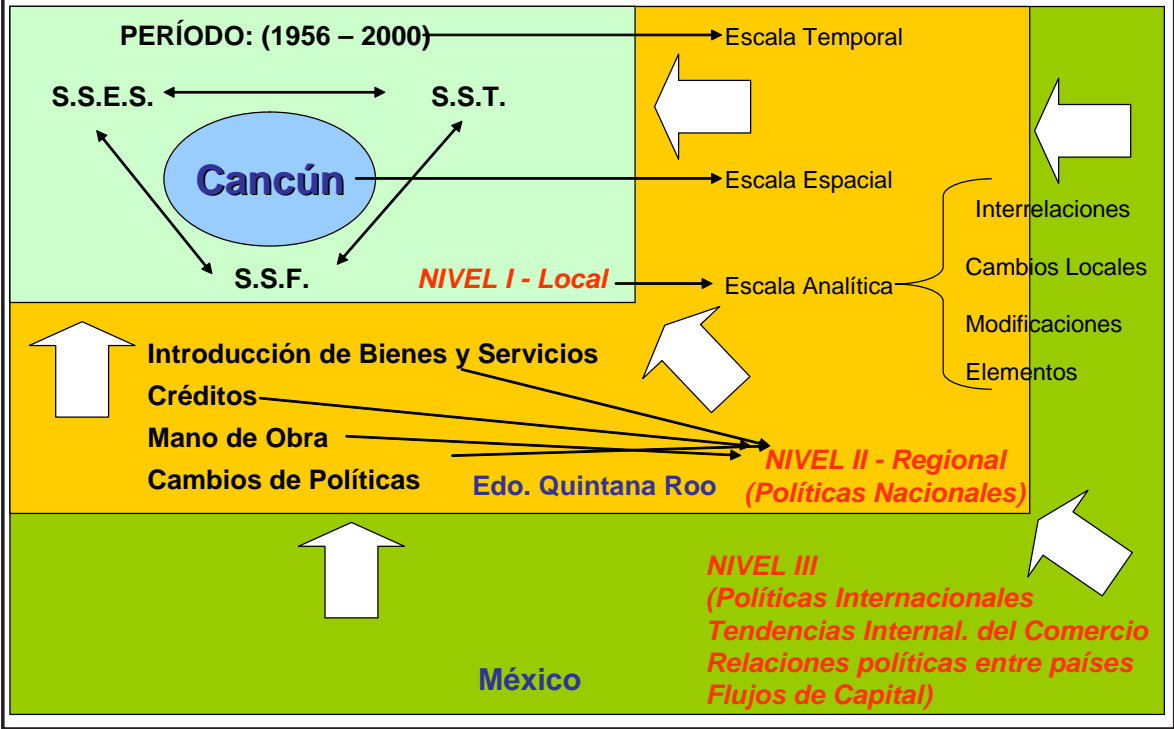

Fuente: Elaboración propia con base en García, 1994. 


\section{EVOLUCIÓN DEL SISTEMA}

Como se ha señalado, la estructura de un sistema complejo queda definida, auto-organizada, cuando pueden establecerse las relaciones entre los subsistemas que lo componen. En este caso, interesa analizar las vinculaciones entre el Sub-Sistema Socioeconómico (SSSE) y el Sub-Sistema Turístico (SST) a lo largo de los períodos identificados.

\section{Primer período (1956-1970): Las condiciones previas para el despegue}

Nivel III: Al terminar la Segunda guerra mundial, la situación de la industria mexicana se deteriora, a consecuencia de la disminución de las exportaciones. El gobierno decide desarrollar el país a través de la industrialización y es necesario buscar un producto que pueda exportarse y que permita financiar las inversiones que se realicen con aquel propósito (...). Así, el turismo surge como el fenómeno económico que puede permitir la obtención de las divisas necesarias para adquirir los bienes de capital que requiere la industrialización (Jiménez, 1984: 44). Igualmente, organismos internacionales como la ONU, el Banco Mundial y el FMI apoyan estas políticas de crecimiento económico (Lanfant, 1980).

El Estado, como parte del proceso de expansión del sector moderno, pone a disposición de los particulares, tanto nacionales como extranjeros, capitales para la activación del sector hotelero, al mismo tiempo de ejecutar una política de re-ubicación de los campesinos propietarios de terrenos ubicados en destinos turísticos estratégicos y apropiarse de los mismos para beneficios particulares de los representantes del gobierno de la época. La deuda pública se incrementa.

Como resultado, toda la red de carreteras y la infraestructura turística nacional se amplió. Así, el "milagro mexicano" fue muy favorable para diversificar la producción nacional e impulsar la actividad turística, tanto en la oferta como la demanda.

A nivel internacional se inicia el auge turístico y el inicio del turismo masivo. Por otra parte, en la lucha por la hegemonía ideológica, la Revolución Cubana pone fin al principal destino turístico norteamericano en el Caribe.

A nivel nacional, aún en medio de una grave crisis social, el presidente Gustavo Díaz Ordaz se dio tiempo para encargar al Banco de México, en 1968, un Plan Nacional de Turismo. Ese plan tenía el objetivo de contribuir al crecimiento del Producto Nacional y al equilibrio en la balanza de pagos que se financiaban con excesivos créditos del exterior, además, el Plan debía generar oportunidades de inversión para el sector privado, crear empleos, alcanzar la autodeterminación y la comercialización de la oferta turística 
nacional en el exterior y lograr la autonomía tecnológica en los servicios turísticos. Dichos objetivos estaban alineados con las recomendaciones que proponía la UIOOT (Antecesora de la actual Organización Mundial del Turismo) para promover el turismo en el mundo (Cancun La Historia, 2005).

Nivel II: Durante este período, existen características muy específicas que permiten afirmar que el sistema considerado era dinámicamente estable. La política económica siguió orientada a dar facilidades a la inversión privada, principalmente por medio de la protección arancelaria, subsidios, exenciones de impuestos, control oficial de las organizaciones obreras, control salarial, liberación de precios, etc. (Méndez, 2000). De esta forma, la industria turística, principalmente los hoteles, se vieron ampliamente beneficiados, aunque las empresas transportistas también supieron aprovechar las oportunidades presentadas.

Debido a la preocupación gubernamental por la actividad turística, se desarrolla una actividad estatal sin precedentes y se consigue la consolidación e institucionalización de la actividad con una Ley de Turismo (Mac Donald, 1981). Además, considerando que la actividad turística requería en ese tiempo de un nivel académico superior para un mejor aprovechamiento de la misma, en este período se establece en la Universidad Autónoma del Estado de México la carrera de técnico en Turismo.

Con base en los lineamientos del presidente Gustavo Díaz Ordaz, el Banco de México creó en 1969 el INFRATUR, para llevar al cabo un Programa Integral de Centros Turísticos. De esa forma, se iniciaron los estudios tendientes a identificar las zonas propicias para la ejecución de proyectos de infraestructura turística, y Cancún y Zihuatanejo fueron seleccionados como prioridades de inversión.

El tema del turismo se convirtió en tema diplomático, ya que otorga estatus a México, junto con una imagen de modernidad. Las Olimpiadas del 68 y el Mundial de Fútbol en 70 se argumentan como eventos aprovechables para dar impulso al turismo. En esta misma época ocurre la masacre de Tlatelolco (1968).

El turismo es considerado como un instrumento de apoyo económico complementario, pero no se debía depender de esta actividad en ninguna de las zonas del país. Sin embargo, por razones económicas, sociales y políticas, se decide impulsar estudios sobre el desarrollo del polo turístico de Cancún.

Nivel I: Quintana Roo apenas tenía poco más de 40,000 habitantes, concentrados en Chetumal, Cozumel e Isla Mujeres. Los censos de aquella época no le daban importancia a la isla de Cancún, habitada sólo durante algunas temporadas del año por un grupo de pescadores. Este pequeño poblado está próximo al despegue, sin embargo en el destino 
turístico más cercano -Cozumel-, la política turística nacional impulsa la construcción de los primeros grandes hoteles de la isla (Figura $n^{\circ} 2$ ).

Figura N²2. Primer Período (1956-1970)

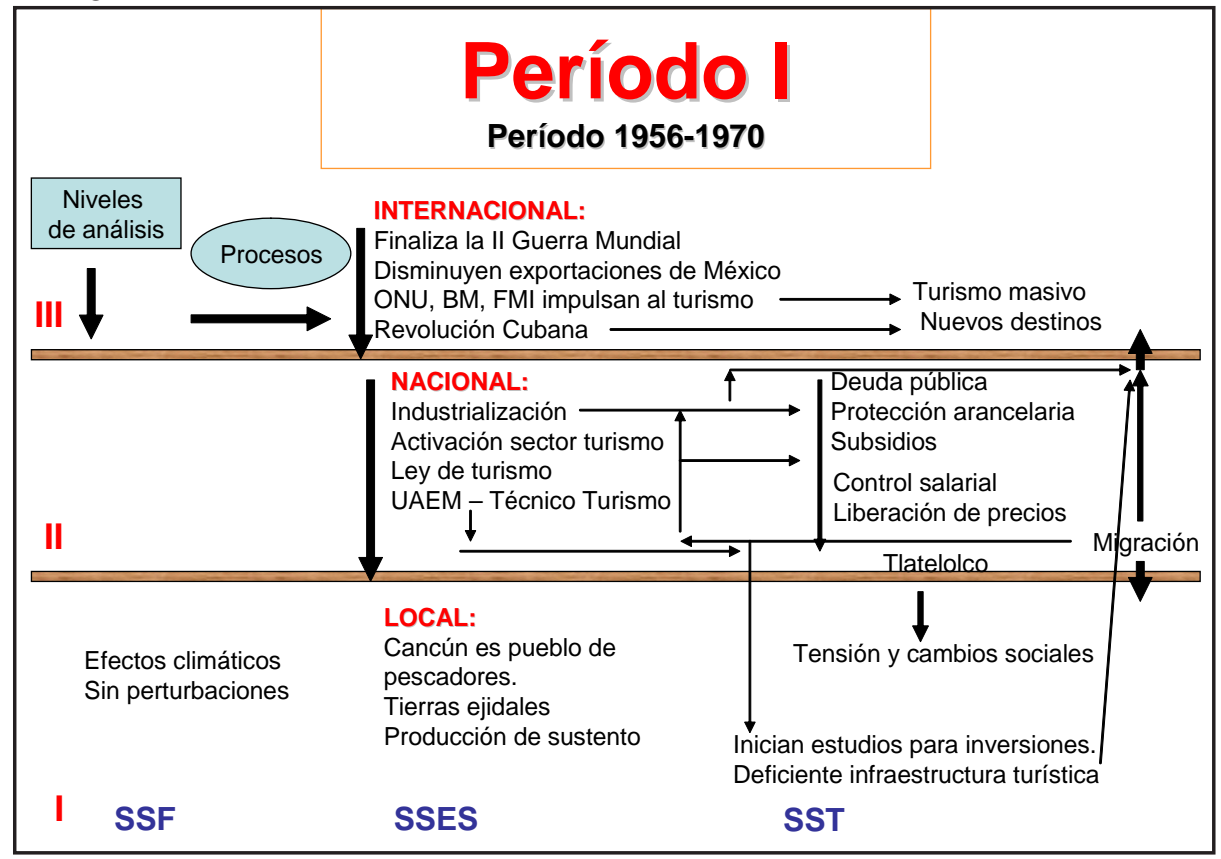

Fuente: Elaboración propia con base en García, 1994.

\section{Segundo período (1971-1976): El proceso de despegue}

Nivel III: Las condiciones externas e internas estaban dadas para iniciar un fuerte impulso a la inversión en turismo: existían los órganos de financiamiento internacional, BM y BID, mientras que en el gobierno federal planeaba los nuevos Centros Integralmente Planeados.

Por otra parte, el desempleo, la pobreza, las desigualdades sociales, etc., impuestas por el modelo económico ejercido, se empiezan a manifestar fuertemente y se hace necesario un cambio profundo en la política económica. Así se pasa del "desarrollo estabilizador" al "desarrollo compartido". Este cambio determina que a partir de ahora el Estado se adjudicará la función de desarrollar los nuevos centros turísticos (macroproyectos) con la intención de mejorar la competitividad en el mercado 
internacional y elevar la captación de divisas.

Nivel II: Se concebía la necesidad de ocupar el nuevo territorio (Quintana Roo) mediante actividades productivas que generaran empleo y reforzaran al sector externo de la economía. Se promueve al turismo como una alternativa viable para utilizarlo como instrumento de desarrollo.

El turismo fue utilizado como el instrumento para absorber a la masa de trabajadores poco calificada o no calificada que iba surgiendo del gran crecimiento demográfico, además de tender un puente entre los ricos y pobres, debido a la radicalización de las posturas ideológicas y las reivindicaciones sociales. El turismo podía incorporar rápidamente a personal poco calificado, con un costo de inversión mínima del Estado.

Nivel I: En Cancún, se inicia la construcción del nuevo polo de desarrollo económico y social, basamentado en la actividad turística, tratando de crear una base económica y fiscal, y un atractivo poblacional para el nuevo Estado de Quintana Roo. Como parte de este plan, se trajeron agricultores de otros Estados de la república, para establecer la infraestructura agrícola de apoyo al consumo turístico (Figura ${ }^{\circ} 3$ ).

A partir del inicio de operaciones de los primeros hoteles en Cancún entre 1973 y 1974, comienza el auge de este destino turístico y paralelamente la consolidación de Quintana Roo como entidad federativa.

El 8 de octubre de 1974, el Congreso de la Unión a iniciativa del presidente Luis Echeverría decreta la creación de los estados número 30 y 31, Quintana Roo y Baja California Sur. Para Quintana Roo fue designado como primer gobernador del estado el Lic. Jesús Martínez Ross (Bautista, 1993). 
Figura N³. Segundo Período (1971-1976)

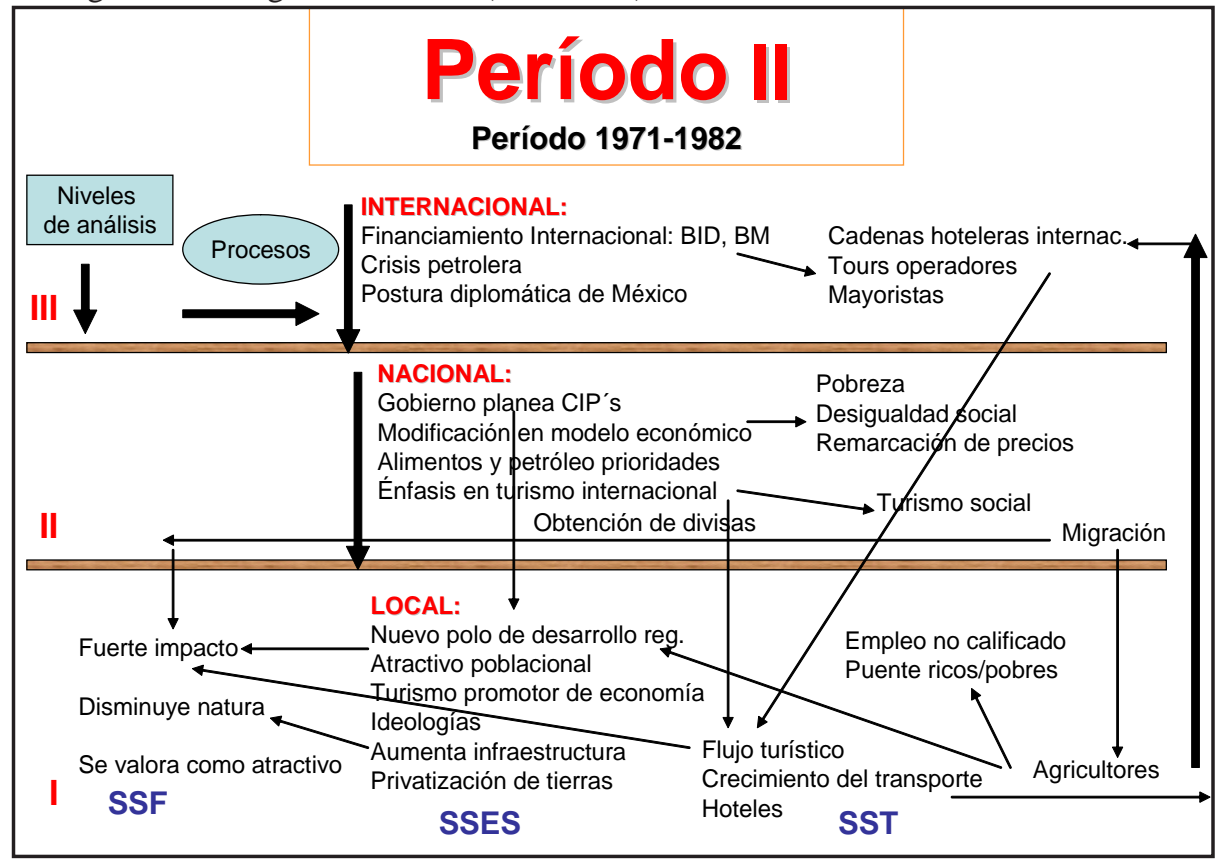

Fuente: Elaboración propia con base en García, 1994.

\section{Período de transición (1976-1982): Estancamiento con inflación}

Nivel III: El régimen iniciado el $1^{\circ}$ de diciembre de 1976, tenía como objetivo fundamental conciliar al Estado con el sector privado. Las intenciones de resarcir al sector privado por los agravios de que había sido objeto durante el gobierno de Echeverría quedaron manifiestos en La Alianza para la Producción, en la que López Portillo propone al sector turismo la creación de 97 mil nuevos cuartos (de los que se han creado 27 mil hasta finales del 2005), con lo que se incrementará paralelamente la demanda de las instalaciones y se dará apertura a la creación de más empleos.

La situación económica y política externa como interna se ve afectada a partir de los años 70 y, en 1977 se inicia el agravamiento de la crisis económica internacional y la postura diplomática de México en el aspecto político, generándose una gran fragilidad en el sector turístico. 
Las cadenas hoteleras internacionales, los tour-operadores y mayoristas, tanto norteamericanos como europeos, comienzan a interesarse en el nuevo destino turístico mexicano, recién creado en Quintana Roo.

Nivel II: En el año 1977 se presenta con una segunda remarcación de precios. Los alimentos y el petróleo se vuelven la prioridad nacional, aunque el turismo se sigue viendo como productor de empleos y factor de desarrollo regional. Así, en 1980, al caer la demanda internacional por el destino mexicano, se comienza a prestar atención al turismo nacional, con una política de turismo social. También se intensificó el fenómeno de la migración del campo a la ciudad.

Nivel I: Se termina la primera fase del megaproyecto Cancún. Se produce un fuerte impacto en el aspecto natural y demográfico debido al flujo turístico y la migración. El pequeño puerto de pescadores va desapareciendo, ante el crecimiento de las empresas vinculadas al turismo. Casi al mismo tiempo, surgen los primeros cinturones de pobreza alrededor de la zona turística.

\section{Tercer período (1983-actual): El camino hacia la madurez}

Nivel III: En los ochenta, México sufría una aguda crisis económica con una alta tasa de inflación, déficit fiscal sin precedentes, carencia de ahorro interno, la reserva internacional de divisas se agotaba, la contracción general del empleo y las quiebras de empresas eran notorias, entre muchos otros problemas. La altísima deuda externa obligaría a la celebración de negociaciones con los acreedores en una posición de debilidad y a la implementación de políticas neoliberales promovidas por el FMI. La racionalidad y reducción del gasto afectó muchas de las actividades que en ese momento se planteaban como importantes para el análisis y seguimiento de las actividades turísticas en el país. Así, la política turística de México en los ochentas, estaría definida por la crisis económica del país y también sería usada como un instrumento de planificación económica para la solución de estos problemas, pero aun así los objetivos básicos eran los mismos: creación de efectos inmediatos en el entorno económico, búsqueda del crecimiento regional, creación de empleos y obtención de divisas fuertes (Jiménez, 1992).

Nivel II: Durante los sesenta y los setenta, el Estado había sido el encargado de impulsar el crecimiento turístico en el país, siendo uno de sus principales inversionistas, sin embargo a partir de 1985 aproximadamente, se verifica un cambio en la política turística, donde el Estado inicia un proceso de des-inversión y progresivamente se va deshaciendo de sus activos y promoviendo las empresas y acciones privadas.

A pesar de la crisis en esta década, la inversión privada nacional e internacional, 
fluyó en cantidades importantes hacia Cancún, aunque luego la crisis financiera detuvo esta corriente inversora.

En el estado de Quintana Roo, las posibilidades laborales de obtener un trabajo de baja calificación y una política de permisibilidad de asentamientos urbanos, estimula la migración intra-estatal.

A nivel estatal, la consolidación estatal de la vocación turística se determina con la creación de la región turística denominada "Riviera Maya", que surge con el surgimiento del municipio de Solidaridad (la cabecera municipal es Playa del Carmen) en 1999 y que comprende un espacio geográfico de Punta Allen a Puerto Morelos. De esta forma se pretende complementar el atractivo natural de Cancún con el atractivo cultural de la región maya.

Nivel I: El crecimiento de la oferta hotelera dio como resultado la necesidad de atraer turismo masivo al nuevo destino, por lo que localmente se comenzaron a privilegiar a los grandes mayoristas internacionales, ignorando a los pequeños empresarios nacionales, quienes también se iniciaban con la actividad turística.

La competencia llegó a niveles de sobreoferta, sobre todo en la industria hotelera, llegando a la necesidad de reducir sus tarifas. Esta situación se agudizó por el impacto del Huracán Gilberto (1984), que puso en crisis de sobrevivencia al destino, llegando al punto de reposicionar a Cancún como destino de clase media, mientras que previamente era un destino de clase alta.

Por su parte, la población local, los migrantes y, los propios turistas, aumentaban la presión urbanizadora y de servicios públicos, generándose de esta forma verdaderos ghettos con trabajadores migrantes hacinados, sin agua ni electricidad, alrededor de un centro turístico internacional integralmente planeado (Figura 4).

El mismo fenómeno de los cinturones de pobreza y colonias totalmente marginadas de los servicios públicos básicos se presentó en los diversos destinos turísticos de la Riviera Maya. 
Figura $\mathrm{N}^{\circ}$ 4. Tercer Período (1983-actual)

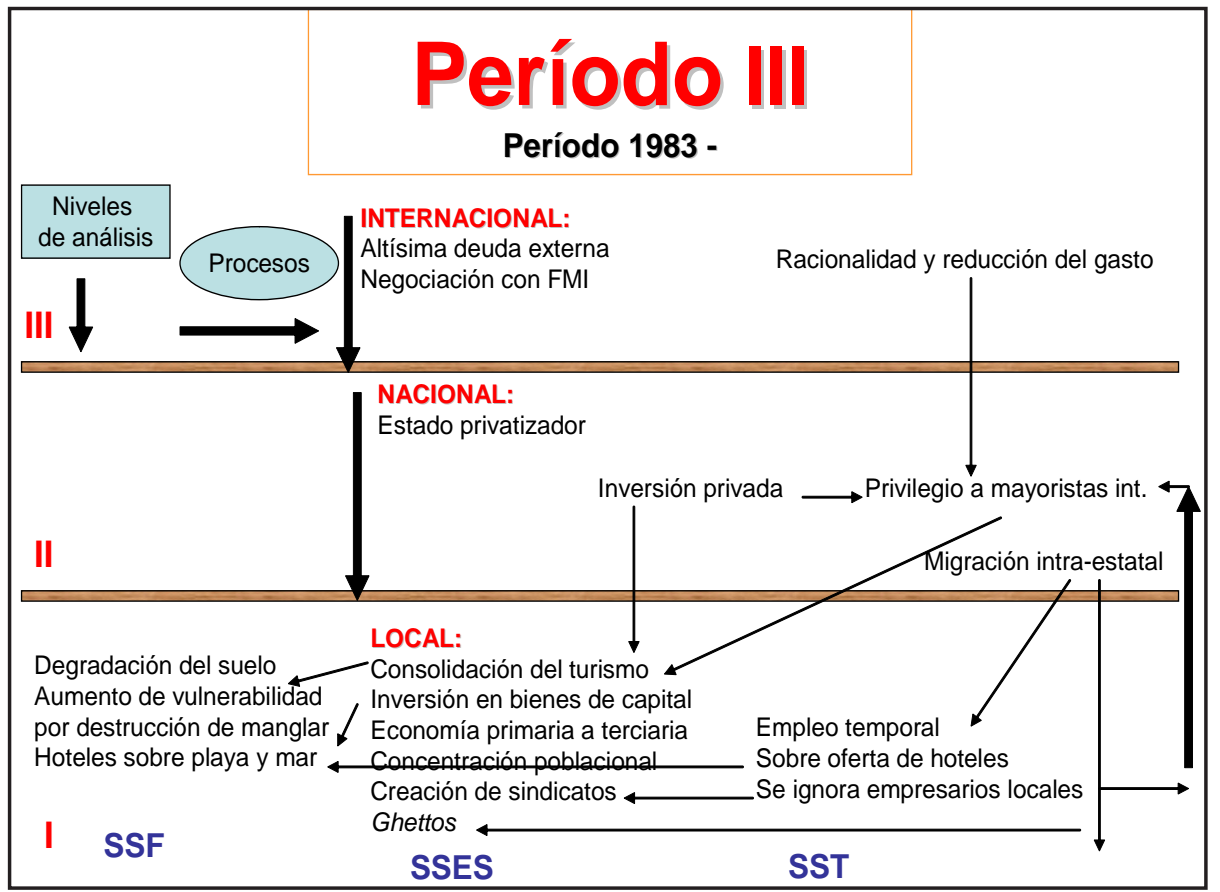

Fuente: Elaboración propia con base en García, 1994.

La presión poblacional y especialmente de la actividad turística propicia que en los instrumentos de planificación turística repetidamente se haga mención a la necesidad de conservar, cuidar y preservar, más que a la intención de aprovechar o utilizar los recursos.

\section{DINÁMICA DEL SISTEMA}

Una vez distinguidos los procesos relevantes y diferenciados los niveles de análisis, se describe el funcionamiento del sistema, su cambio de un período a otro, su desestabilización y reorganización con una nueva estructura.

La descripción del sistema y su dinámica permitió determinar que existen fluctuaciones en todos los niveles de análisis, que tienen un cierto nivel de vulnerabilidad (umbral de tolerancia a las perturbaciones, pasado ese límite, el sistema se desestabiliza) e impredecibilidad (el sistema va a derivar a una nueva situación por: a) una dependencia sensitiva de las condiciones iníciales, b) alta sensibilidad a ciertas situaciones) y que 
distinguen un período de otro, dentro del mismo sistema. En este sentido, durante el Primer período analizado, la vulnerabilidad se presentó por una combinación de los siguientes factores: a) La industrialización acelerada del país (Nivel II); b) El desarrollo de la frontera Sur (Nivel II); c) El incremento importante de la deuda pública (Nivel II); d) Conflictos sociales a nivel nacional e internacional (Nivel III).

Bajo estas condiciones y con estos factores presentes, el sistema ya no pudo retornar a su estado original, por lo que evolucionó a un Segundo período con nuevos factores presentes: a) El aprovechamiento de la actividad turística (Nivel II), que se traduce en la creación de Cancún (Nivel I); La protección de la industria nacional (Nivel II) para ocupar mano de obra; c) Cuantiosos préstamos externos (Nivel II), utilizados en parte para seguir ampliando la red carretera y; d) Creación de varias leyes de carácter social y educativo (Nivel II), tratando de disminuir la presión social. En Cancún (Nivel I), los primeros turistas internacionales de clase alta, comienzan a arribar al recién creado destino turístico.

A nivel nacional (III), debido al cambio de presidente se promueve un resarcimiento hacia el sector privado, que había sido agraviado por el régimen anterior. Se inicia una Transición con las siguientes características: a) México se convierte en potencia petrolera (Nivel II) al mismo tiempo que se abandona el sector agropecuario (Nivel II) y b) se alienta la actividad turística de playa (Nivel I). c) Se recurre al endeudamiento externo (Nivel II) para seguir apoyando la política proteccionista, lo cual a su vez genera una sobre-valuación del peso que permite un turismo emisivo de proporciones considerables. Se publica la Ley de Inversiones Extranjeras (Nivel III) y esto se refleja en Cancún (Nivel I), donde las empresas hoteleras realizan grandes inversiones.

Ya en el tercer período, el auge económico (1979-1981) propició un extraordinario repunte del empleo, incorporando amplios contingentes de mujeres en la industria de transformación y de servicio (Niveles II y I). Finalmente, en 1982 el Estado Protector llega a su fin por dos fenómenos económicos a considerar; uno de naturaleza interna (Nivel II), la crisis del modelo económico a través de la sustitución de importaciones y el proteccionismo económico; y otro de origen externo (Nivel III), la difusión del neoliberalismo y su complemento: la teoría monetarista del FMI y el Banco Mundial.

También durante estos años, el turismo egresivo (Nivel II) crece rápidamente, mientras que los centros turísticos nacionales (Nivel I) tienen problemas para lograr las tasas de ocupación adecuadas debido a diversos factores. Entonces se dedica atención al Plan Nacional de Turismo en donde se pretende retener a los mexicanos e incentivarlos a que realicen turismo interno, entre ellos al nuevo destino nacional: Cancún (Nivel I). 
En este período, durante el año 1984, el huracán Gilberto (Nivel I) azota esta ciudad y determina el cambio de segmento de mercado, demostrando la vulnerabilidad e impredecibilidad del destino turístico.

Después de esto, el gobierno nacional (Nivel II) apoya a Cancún (Nivel I) para que el sistema sigua su marcha o transición hacia un Cuarto período, que se presenta dentro de los instrumentos de política pública bajo la forma del desarrollo sustentable para las comunidades anfitrionas, por medio de la aplicación de acciones concretas surgidas de las recomendaciones de la Agenda 21 Local.

La transición se consolida con la preocupación por la seguridad y protección al turista, luego del ataque terrorista a la ciudad de Nueva York, el 11 de septiembre del 2001, y el surgimiento de la gripe humana AH1N1, que surge en mayo de 2009.

A nivel local, el crecimiento acelerado de la Riviera Maya impulsa a la ciudad de Tulum a convertirse en municipio independiente en marzo de 2008, separándose del municipio de Solidaridad, del cual formaba parte.

\section{CONCLUSIONES}

Los diversos instrumentos de política turística analizados son prueba de la presencia de una política pública de orden superior que además se sustenta y fundamenta teórica e ideológicamente en paradigmas que trascienden los límites estatales o nacionales. Los documentos públicos muestran confluencia entre los contenidos expresos en cada uno de ellos con la política turística nacional e internacional en dos grandes contextos: el económico social y el físico o territorial, los cuales se sobreponen y fungen como marco en el cual se han elaborado y desarrollado las diversas estrategias, tácticas y acciones turísticas, con esto se confirma el estrecho acoplamiento que de manera deliberada se plantea desde el nivel local hacia el global.

En el contexto económico social se puede observar el cambio de dirección en el modelo económico del país desde una economía orientada a la sustitución de importaciones hacia una economía neoliberal a de la década de los noventa. En el estado de Quintana Roo, la planificación turística dejó de centrarse exclusivamente en la promoción turística de Cancún y se amplió hacia la la Riviera Maya por medio de la apertura a la inversión extranjera, estableciendo los mecanismos y posibilidades para impulsarla. Los instrumentos de planificación turística orientaron la inversión hacia el nuevo proyecto de la "Riviera Maya", que se dio de forma coincidente con el cambio de discurso político hacia la descentralización de funciones pública, la diversificación de la actividad turística, y las recomendaciones sobre la sustentabilidad del turismo. 
En el contexto socio económico de la multiculturalidad, debido a las migraciones inter-estatales, la presencia de turistas-residentes, los “mestizos” y la población maya, ha propiciado una coexistencia y convivencia de contrastes muy amplios. Esta misma situación se ve reflejada en la economía y los instrumentos de política turística analizados, en los que muy diversas intenciones y propuestas se ven planteadas, pero pocas de ellas se ven cristalizadas, apareciendo nuevas intenciones al entrar en vigor cada nuevo plan. De esta forma, Cancún en la actualidad presenta las características propias de un sistema complejo, donde la cooperación entre las partes determina la sinergia y cuyo sistema total no es descomprimible, ya que los múltiples factores locales, estatales y nacionales no pueden ser aislados y cuya realidad actual sólo puede ser explicada por medio de un análisis totalizador.

El análisis totalizador, centrado en la descripción de los cambios estructurales en el sistema, es una forma viable de asomarse a la complejidad, aunque esto suponga que al mismo tiempo se dejen de observar otras posibilidades de las relaciones en los sistemas, es decir, si bien es una mirada a la complejidad, ésta, la complejidad se mantiene como característica del sistema. Se debe acotar asimismo, que el hecho de que se hayan presentado ciertos cambios en el pasado de un sistema complejo, de ninguna manera pueden proponerse como condiciones para futuros cambios, así como tampoco significa que los cambios observados puedan repetirse bajo condiciones similares en otro sistema turístico, cada sistema, mantiene una identidad propia y un conjunto propio de formas de auto-organizarse. Lo valioso en términos de la comprensión de la complejidad es entonces, la posibilidad que la aproximación metodológica elegida tiene para revelar ciertos aspectos de las relaciones funcionales y estructurales entre sistemas, subsistemas y niveles de observación de los mismos.

Aceptar que un sistema turístico presenta un comportamiento comprensible sólo como un sistema complejo, implica el reconocimiento de que existen: relaciones ocultas entre sus elementos y hacia su entorno, las cuales pueden permanecer así o hacerse evidentes en cualquier momento del tiempo, que tales relaciones involucran procesos que por mínimos o pequeños que sean pueden desencadenar una transformación total del sistema bajo determinadas condiciones, que existe un acoplamiento funcional y estructural del sistema con otros sistemas y con su entorno, que a su vez son dinámicos y tienden a su vez a reorganizarse y adaptarse a su propio entorno. Lo anterior, llevado a la práctica del diseño y ejecución de políticas turísticas, por ejemplo, o al terreno de la planificación turística tiene consecuencias evidentes: a) toda política o ejercicio de planificación debe considerar los procesos que desempeñan las relaciones principales entre los elementos del sistema como marco inicial de aplicación, b) toda acción en este campo debería incluir a la incertidumbre como posibilidad, traducida en un análisis de riesgo asociado a cualquier política o plan, c) todo ejercicio de planificación debe considerar el acoplamiento que el sistema turístico muestra hacia otros sistemas, 
particularmente los económicos y políticos de orden nacional, internacional y global y d) se deben diseñar estrategias de vigilancia/observación de la operación del sistema de modo que sea posible identificar comportamientos contingentes, resultados imprevisibles de las decisiones tomadas.

\section{BIBLIOGRAFIA}

- Ander-Egg, Ezequiel (2001). Metodología y práctica del desarrollo de la comunidad. Editorial Lumen. Buenos Aires, Argentina.

- Bar-Yam, Yaneer (1997). Dynamics of Complex Systems (Studies in Nonlinearity). Editorial Perseus Books Group. Cambridge, USA.

- Becerra, N.; et al. (1997), Un análisis sistémico de políticas tecnológicas. Estudio de caso: El agro pampeano argentino 1943-1990, Centro de Estudios Avanzados, Universidad de Buenos Aires. Bs. As., Argentina.

- Cordera, Rolando (1979). Estado y Economía en México: La Perspectiva Histórica. Editorial CIDE. Economía de América Latina. № 3. México, D.F. p 101-123.

- Dobb, Maurice. (1991). Estudios sobre el Desarrollo del Capitalismo. Editorial Siglo XXI. México, D.F.

- García, Rolando (1994). Interdisciplinariedad y sistemas complejos, en Enrique Leff y otros. Ciencias Sociales y formación ambiental. Editorial Gedisa. Barcelona, España

- García, Rolando (2000). El conocimiento en construcción. Editorial Gedisa. Barcelona, España.

- Gunn, Clare (1994) Tourism Planning: Basic, Concepts, Issues. Taylor and Francis. Washington, EUA.

- Holder, J. (1987). “The Patterns and Impact of Tourism” en Environmentally Sound Tourism in the Caribbean. The Banff Centre School of Management, Abril 1987. Barbados.

- Iracheta, Alfonso (1992). Hacia una Planeación Urbana Crítica. Editorial U.A.E.M. Toluca, México.

- Jiménez, Alfonso (1984). Turismo Estructura y Desarrollo. Editorial InterAmericana. México, D.F.

- Jiménez, Alfonso (1992). Turismo Estructura y Desarrollo. Editorial McGraw Hill. México, D.F.

- Jiménez, Alfonso (2005) Una aproximación a la conceptualización del turismo desde la teoría general de sistemas. Universidad del Caribe y M. A. Porrúa, México.

- Lanfant, Marie (1980). El turismo en el proceso de internacionalización. UNESCO. Revista Internacional de Ciencias Sociales Nº. 1, Vol. XXXII. Francia, París. p 1444.

- Lanfant, Marie (1995). International tourism: identity and change. Editorial Sage. 


\section{California, USA}

- Leff, Enrique (1986). Racionalidad ambiental, Productividad Ecotecnológica y manejo integrado de recursos. Ecología y capital - Racionalidad ambiental, democracia participativa y desarrollo sustentable. Editorial Siglo XXI. México, D.F. p 236-257.

- Luhmann, Niklas (1997). Sociedad y Sistema: la ambición de la teoría. Editorial Paidós/I.C.E.-U.A.B. Barcelona, España.

- Mac Donald, Eugenio (1981). Turismo: Una Recapitulación. Editorial Bodoni. México, D.F.

- Méndez, José (2000). Problemas Económicos de México. Editorial McGraw Hill. México, D.F.

- Mill, Robert Y Morrison, Alastair (1985) The tourism system: An introductory text. Prentice Hall. Englewood Cliffs, EUA.

- Morin, Edgar (1995). Introducción al Pensamiento Complejo. Editorial Gedisa. Barcelona, España

- Osorio, Maribel (2010) Turismo masivo y alternativo. Distinciones de la sociedad moderna/posmoderna. En Convergencia, Revista de Ciencias Sociales, Núm. 52, enero-abril 2010, Toluca, México.

- Rodríguez, Gerardo (1999). Hacia una epistemología integral. Editorial UAEM. Toluca, México.

- Romo, Héctor G. (1995). Orígenes de la crisis en México. Editorial Era. México, D.F.

- Rostow, Walt (1961). Las Etapas del Crecimiento Económico. Editorial F.C.E. México, D.F.

Recibido: 28/11/2007

Devuelto para correcciones: 23/04/2008

Aprobado:15/07/2010

Arbitrado anonimamente 